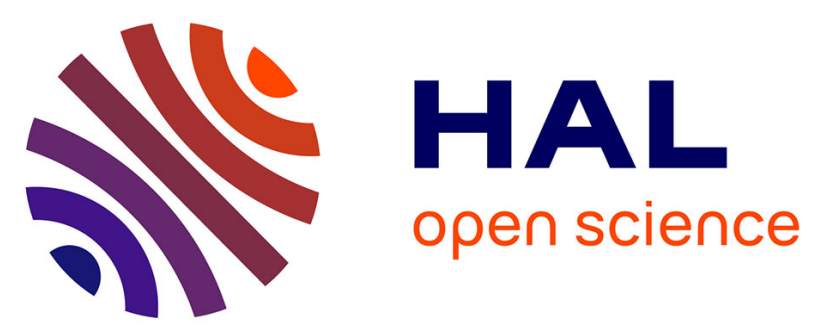

\title{
One-shot measurement of the three-dimensional electromagnetic field scattered by a subwavelength aperture tip coupled to the environment
}

Nancy Rahbany, Ignacio Izeddin, Valentina Krachmalnicoff, Rémi Carminati, Gilles Tessier, Yannick de Wilde

\section{To cite this version:}

Nancy Rahbany, Ignacio Izeddin, Valentina Krachmalnicoff, Rémi Carminati, Gilles Tessier, et al.. One-shot measurement of the three-dimensional electromagnetic field scattered by a subwavelength aperture tip coupled to the environment. ACS photonics, 2018. hal-02360079

\section{HAL Id: hal-02360079 \\ https://hal.science/hal-02360079}

Submitted on 12 Nov 2019

HAL is a multi-disciplinary open access archive for the deposit and dissemination of scientific research documents, whether they are published or not. The documents may come from teaching and research institutions in France or abroad, or from public or private research centers.
L'archive ouverte pluridisciplinaire HAL, est destinée au dépôt et à la diffusion de documents scientifiques de niveau recherche, publiés ou non, émanant des établissements d'enseignement et de recherche français ou étrangers, des laboratoires publics ou privés. 


\title{
One-shot measurement of the three-dimensional electromagnetic field scattered by a subwavelength aperture tip coupled to the envi- ronment
}

\author{
Nancy Rahbany ${ }^{\dagger}$, Ignacio Izeddin ${ }^{\dagger}$, Valentina Krachmalnicoff ${ }^{\dagger}$, Rémi Carminati $^{\dagger}$, Gilles Tessier $^{* \ddagger}$, \\ Yannick De Wilde*†
}

†ESPCI Paris, PSL Research University, CNRS, Institut Langevin, 1 rue Jussieu, F-75oo, Paris, France

‡Sorbonne Université UPMC Univ. Paris 06, Institut de la Vision, INSERM UMR_S968 CNRS UMR 7210, and CNRS

UMR 8250 Neurophotonics laboratory, 45 Rue des Saints Pères, 75006 Paris, France

KEYWORDS. Near-field scanning optical microscopy, digital holography, complex electromagnetic field reconstruction, angular radiation pattern, leaky surface plasmons

\begin{abstract}
Near-field scanning optical microscopy (NSOM) achieves subwavelength resolution by bringing a nanosized probe close to the surface of the sample. This extends the spectrum of spatial frequencies that can be detected with respect to a diffraction limited microscope. The interaction of the probe with the sample is expected to affect its radiation to the far field in a way that is often hard to predict. Here we address this question by proposing a general method based on full-field off-axis digital holography microscopy which enables to study in detail the far-field radiation from a NSOM probe as a function of its environment. A first application is demonstrated by performing a three-dimensional ${ }_{3} \mathrm{D}$ ) tomographic reconstruction of light scattered from the sub-wavelength aperture tip of a NSOM, in free space or coupled to transparent and plasmonic media. A single holographic image recorded in one shot in the far field contains information on both the amplitude and phase of the scattered light. This is sufficient to reverse numerically the propagation of the electromagnetic field all the way to the aperture tip. Finite Difference Time Domain (FDTD) simulations are performed to compare the experimental results with a superposition of magnetic and electric dipole radiation.
\end{abstract}

Near-field Scanning Optical Microscopy (NSOM) has proven to be a powerful tool for investigating the behavior of light at distances from the sample much smaller than its wavelength $\lambda^{1}$. NSOM uses a scanning probe with a sub- $\lambda$ sized scatterer to measure the high frequency spatial components of the electromagnetic (EM) field, contained in the evanescent waves which are confined in the near field. This allows one to perform sub- $\lambda$ imaging or to detect purely evanescent plasmonic fields. Conversely, the probe of a NSOM can be used to locally excite surface plasmon polaritons ${ }^{2}$. However, a limitation of this method generally arises from the scarce information on the way the sub- $\lambda$ probe of a NSOM scatters light to the far field, and how it reacts with the environment. Whereas it is worth noticing that surface plasmons excitation can also be achieved by alternative methods such as the use of tunneling electrons ${ }^{3,4}$, pointlike dipoles $^{5}$, or surface defects ${ }^{2,6}$, it is of great importance to develop experimental approaches to characterize the scattering properties of NSOM tips and its coupling with the local environment.

Apertureless metallic NSOM tips in free space are usually modeled as effective electric dipoles producing a field enhancement for the electric field component parallel to the tip axis7,6,8, but the coupling of such scattering tips to the environment is a complex problem that needs to be taken into account. A metal coated sub- $\lambda$ aperture at the extremity of a tapered fiber is a practical realization of a sub- $\lambda$ hole in a perfectly conducting plane screen initially studied theoretically by Bethe 9 , which radiates in free space as a coherent superposition of a magnetic and an electric dipole $e^{6,10-12}$. A generalization of the two-dipole model has also been proposed based on multipole expansion to describe the far-field transmission pattern in free space $^{13}$, while their resolving power as near-field probe was addressed with a model using a ring-like current ${ }^{14}$. Nevertheless, recent near-field optical images of intensity measured on nanoantennas with a metalcoated aperture at the end of a hollow pyramidal probe suggest that the probe essentially behaves as a tangential magnetic dipole when it is coupled to a metallic nanoantenna ${ }^{15}$. Advanced fabrication techniques aim at achieving high control of the optical response of optical nanoantennas as they can be directly installed at the extremity of NSOM probes ${ }^{16,17}$. However, defects play an important role at the nanoscale ${ }^{18}$. Each probe is therefore unique, and its radiation pattern may dramatically differ from expectations. Individual NSOM probe characterization is absolutely necessary to achieve quantitative measurements. Regardless of the type of probe used, placing it on the surface of a sample induces complex reflections and interferences in the EM field between the scattering probe and the surface. This results in a dressed polarizability of the coupled system and a radiation pattern which is difficult to predict, especially if the size of the scatterer is not negligible with respect to $\lambda$ or if the coupling is strong between the scatterer and the sample surface ${ }^{19,20}$. 
There is thus an obvious need to characterize how the EM field is scattered by a nanostructure in a given environment. NSOM is the ideal playground for such a study as it allows to precisely control the coupling between a nanostructured probe and the local environment.

In the past, back focal plane imaging was successfully applied to measure the far-field angular radiation pattern of leaky surface plasmons excited by a NSOM probe on a metallic thin film ${ }^{2,21,22}$. The information regarding the phase of the EM field is inherently lost because the measurement is only based on the mapping of the intensity in the Fourier plane of an optical system ${ }^{16,23}$. In order to infer the EM field in any plane, i.e. in three dimensions, a precise knowledge of both the amplitude and phase of the EM field is absolutely essential.

In this paper, we demonstrate full three-dimensional $(3 \mathrm{D})$ far-field tomographic reconstruction in real space of the light scattered through a metal coated aperture pyramidal NSOM tip using Digital Holographic Microscopy (DHM) ${ }^{24}$. From a single hologram recorded in the far field, both the amplitude and phase of the EM field can be calculated in the measurement plane and then back-propagated numerically towards the source, allowing a complete $3 \mathrm{D}$ representation of the EM field ${ }^{25}$. DHM was previously combined with NSOM for the investigation of the super-resolving capabilities of disordered scattering media assuming that the aperture tip produces point-like illuminating fields ${ }^{26}$. Here, DHM is combined with a NSOM to investigate in details how the light is scattered from its aperture tip when it is either in free space, above a glass surface, or above the surface of a metallic thin film on glass. The $3 \mathrm{D}$ reconstruction of the EM field scattered by the aperture tip in free space shows a broad angular distribution of intensity with a maximum in the forward direction. When the aperture tip is brought in contact with a glass surface, directional lobes at the critical angle of the glass/air interface are superimposed on the broad distribution, while a highly directional supercritical emission is measured when the aperture tip is at the surface of the metallic film on glass due to the excitation of leaky surface plasmons. Finite Difference Time Domain (FDTD) simulations support the approximation of the aperture tip of the NSOM as a superposition of electric and magnetic dipoles.

\section{RESULTS AND DISCUSSION}

Experimental Setup and Methods. Our optical setup is schematically depicted in Figure 1. It is composed of an offaxis DHM combined with a commercial NSOM ${ }^{27}$ modified to allow interferometric measurements (see Measurement section). Light from a single mode $633 \mathrm{~nm}$ He-Ne laser is split into sample and reference beams. The sample beam is focused on the apex of a metal coated aperture probe, which is either in free space or placed above a sample. SEM images of the probe provided by the manufacturer ${ }^{28}$ are shown in Figure 2.d. The sample is mounted on a piezoelectric three-axis translation stage, which allows one to bring its surface in contact with the aperture tip, or to finely adjust the tip height above the sample. The set-up operates in transmission mode with a high numerical aperture microscope objective used to collect the light emerging from the aperture tip. The collected light (through the substrate if any) is directed to a CCD camera where it interferes with the reference beam in an off-axis manner, creating a hologram. The hologram pro- vides a one-shot measurement of the spatial variations of the amplitude and phase, which fully characterizes the wave front in the plane of the CCD camera. The propagation of EM fields is accurately described by diffraction theory by means of the angular spectrum representation ${ }^{6}$. Using backpropagation, the amplitude and phase of the complex EM field at any point in space can thus be reconstructed by computing the scattered field in Fourier space (k-space), provided that the propagation medium is homogeneous.

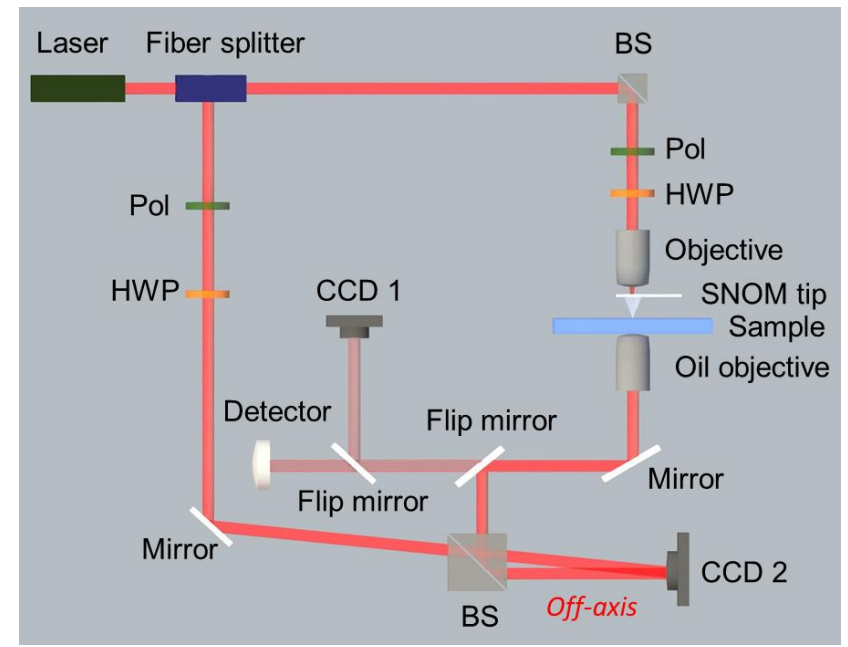

Figure 1. Schematic of the optical setup: an off-axis digital holographic microscope (DHM) is combined with a nearfield scanning optical microscope (NSOM) with aperture metal-coated hollow pyramidal tip. BS: beam splitter, Pol: polarizer, HWP: half-wave plate, CCD: charge coupled device camera.

With this approach, after investigating the radiation by the aperture tip in free space, we investigate the coupling of the tip with two types of samples: a transparent glass sample, and a plasmonic sample made of a $40 \mathrm{~nm}$ gold film deposited on a glass substrate (see Fabrication section). The reconstruction of the EM field scattered by the tip in various environments is achieved numerically after k-space filtering using a method inspired by Cuche et al. ${ }^{24,25}$, as illustrated in Figure 2.

In Figure 2.a., we show a typical hologram, captured at the CCD plane, resulting from the interference between the reference beam and the light scattered through the nanosized aperture of the NSOM tip when it is placed in contact with the plasmonic sample. Interference fringes typical of off-axis holography can be seen in the magnified image in the inset. The reconstruction is performed by first calculating the Fourier transform of the recorded real space hologram. In the kspace representation shown in Figure 2.b., the contributions of the zeroth order of diffraction appear near the center. Two interference terms, which are of interest to reconstruct a real (order +1 ) or a virtual (order -1) image of the EM field as they contain the amplitude and phase information, appear as satellites symmetrically displayed along a diagonal on both sides of the zeroth order term. The separation in k-space results from the off-axis configuration where the sample beam is tilted by a few degrees with respect to the reference beam $^{24}$. Only one interference term is kept after filtering in k-space the Fourier transform of the hologram: the one required to construct the real image, highlighted by a red circle 
in Figure 2.b. If $z$ corresponds to the propagation direction, the spatial Fourier transforms of the EM field in planes with different $z$ values are linked by a simple propagator ${ }^{6}$. Multiplying the filtered Fourier transform of the hologram by the propagator allows one then to calculate the complex EM field in any z-plane after the NSOM tip by a simple inverse Fourier transform. In this way, a $3 \mathrm{D}$ stack of images giving a tomographic reconstruction of the amplitude and phase of the EM field in the vicinity of the NSOM tip can be calculated from the measurement of a single hologram, as depicted in Figure 2.c. In a first approximation, the light source corresponding to this nano-aperture is point-like. The optical intensity therefore decreases very fast, as $\rho^{-2}$, where $\rho$ is the distance to the tip in spherical coordinates. This sharp decrease is difficult to represent, and we have therefore chosen, for clarity, to multiply the intensity maps by $\rho^{2}$. Note that a similar reconstruction can be performed to map the phase of the EM field ${ }^{24}$.
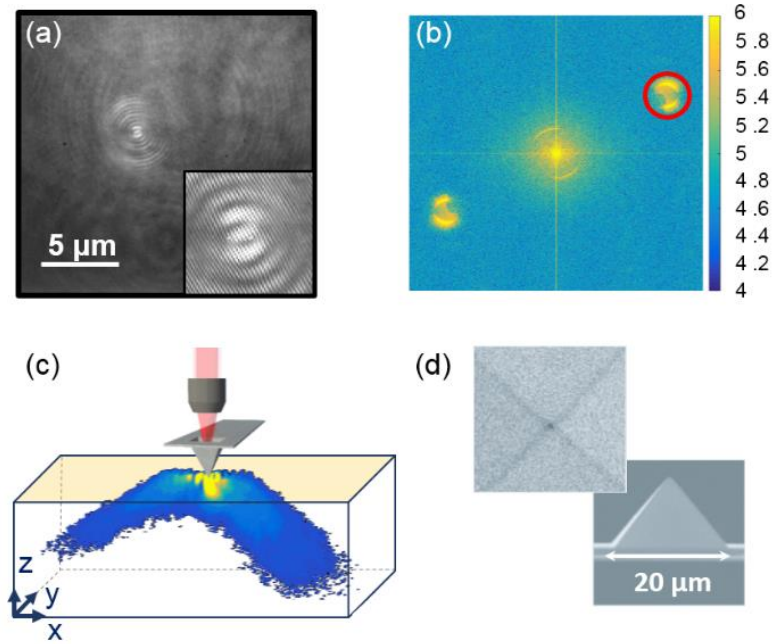

(d)

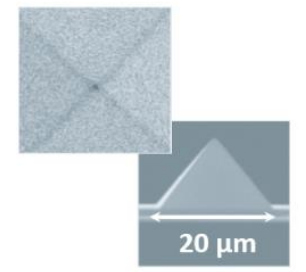

Figure 2. Field reconstruction procedure. (a) Raw hologram of the scattered light from the nanosized aperture of a NSOM tip through a plasmonic sample made of a $40 \mathrm{~nm}$ gold film on a glass substrate. Inset: magnified image showing the interference fringes. (b) Fourier transform of the hologram (a) showing the oth order of diffraction (around the image center) and the two interference terms (corresponding to the real and virtual images) in k-space. The real image term (inside the red circle) is selected by spatial filtering. (c) ${ }_{3} \mathrm{D}$ tomographic reconstruction of the scattered field intensity through the substrate up to a distance of $10 \mu \mathrm{m}$ under the tip. (d) SEM images of the NSOM probe of aperture size $\sim 150$ $\mathrm{nm}^{28}$.

3D Reconstructed EM field through different media. Applying this method, we compare the $3 \mathrm{D}$ radiation patterns of the light scattered by a NSOM tip placed either in free space, in contact with a glass coverslip- a dielectric sample-, or on a metallic thin film on glass. $2 \mathrm{D}$ cross sections of the reconstructed EM field intensities are shown in Figure 3, in the $\mathrm{x}-\mathrm{z}$ plane. This plane is perpendicular to the sample surface $(x-y)$, contains the axis of the linearly-polarized illumination $(\mathrm{x})$, and is perpendicular to the axis of the cantilever (y). As our method captures the complex EM field, we can also represent the wavefront reconstructions (Figures 3.c., 3.g., and 3.k.) obtained by multiplying the amplitude at each point in space $|\mathrm{A}(\mathrm{x}, \mathrm{y}, \mathrm{z})|$ by the cosine of the corresponding phase $\cos \phi(x, y, z)$. We observe that in the freespace case (Figures 3.b. and 3.c.), which gives access to the intrinsic radiation pattern of the aperture tip without any coupling to the environment, light is only scattered forward beneath the tip with a broad angular distribution centered around $\theta=0^{\circ}$. When the tip is in contact with the glass sample (Figures 3.f. and 3.g.), light is still scattered in the forward direction (defined as $\theta=0^{\circ}$ ) but two preferred radiation directions at the critical angle $\pm\left|\theta_{c \text {,glass }}\right|$ also appear as a result of the interaction with the air-glass interface. According to Snell's law, $\left|\theta_{\text {c,glass }}\right|=41.8^{\circ}$. Interestingly, in Figures 3.j. and 3.k., we can clearly see that due to the presence of the gold film, leaky surface plasmons are generated that propagate along the metal surface and radiate into the glass substrate at specific angles, i.e. resonance angles, that satisfy the phase matching condition between surface plasmons at the airmetal interface and photons in glass. This explains the presence of two narrow lobes in each term of the k-space representation of the hologram in Figure 2.b. Since the NSOM tip is illuminated by linearly polarized light, a direction of excitation of the surface plasmons is favored and their wavevector must satisfy the phase matching condition, which results in the two lobes ${ }^{2}$. These lobes correspond to the directional radiation that is clearly observed in the k-space in Figure 2.b., and in the object space in Figures 2.c. and 3.j. and 3.k. In addition, the $x-y$ intensity images presented in Figure $\mathrm{S}_{1}$ (Supporting Information) show that the excited surface plasmons exhibit a double-lobed pattern due to the fact that they can only be excited by p-polarized field components ${ }^{2,6}$.

The resonance angle $\theta_{c, g o l d}$, which is the angle between the transmitted rays and the surface normal, can be calculated according to the conservation of parallel momentum equation and the surface plasmon dispersion relation, as follows:

$$
n_{\text {glass }} \frac{2 \pi}{\lambda} \sin \theta_{c, \text { gold }}=\operatorname{Re}\left\{\frac{2 \pi}{\lambda} \sqrt{\frac{\varepsilon_{\text {gold }}}{\varepsilon_{\text {gold }}+1}}\right\}
$$




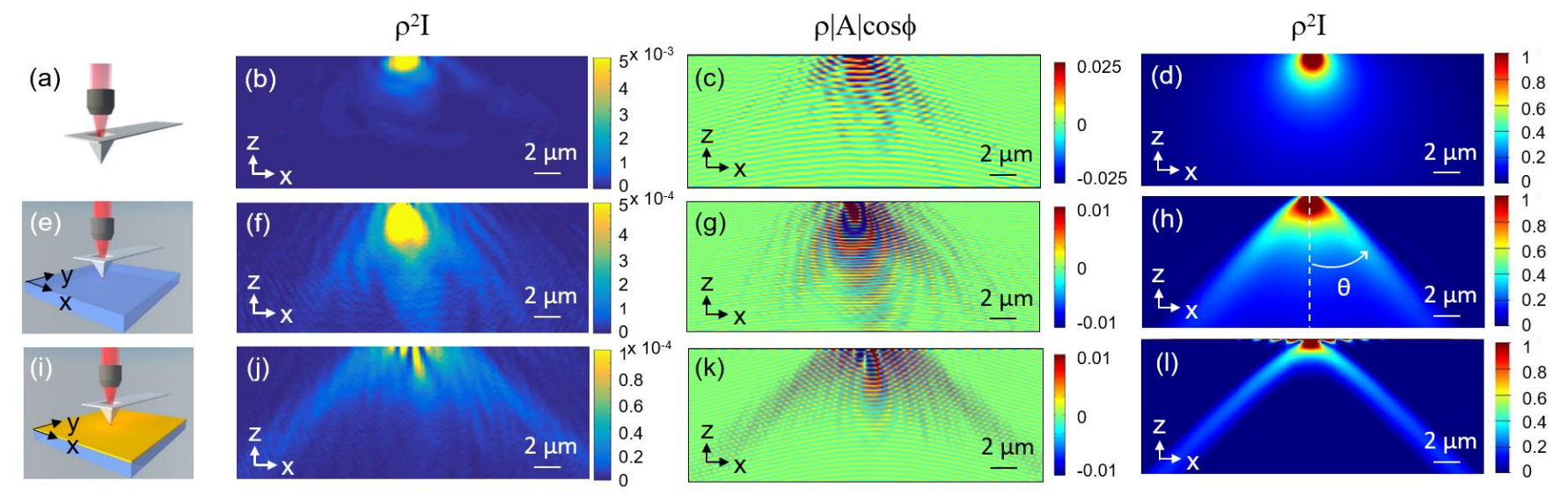

Figure 3. Configuration with the nanosized aperture of a NSOM tip (a) in free space, and in contact with (e) a transparent glass substrate, and (i) a $40 \mathrm{~nm}$ gold film on a glass substrate. (b),(f),(j) are the corresponding $2 \mathrm{D}$ cross sections in the $\mathrm{x}-\mathrm{z}$ plane along the axis of the NSOM tip of the $3 \mathrm{D}$ reconstructed scattered field intensity obtained by off-axis Digital Holographic Microscopy measurement of the complex EM field (in arbitrary units). For better contrast, the intensities have been multiplied by $\rho^{2}$, with $\rho$ the distance to the probe. (c), (g),(k) are the corresponding $2 \mathrm{D}$ cross sections of the experimental reconstructed wavefronts $\rho|A(x, y, z)| \cos (\phi(x, y, z))$, where $A(x, y, z)$ is the amplitude, and $\phi(x, y, z))$ the phase, of the $3 D$ reconstructed complex EM field. The results of FDTD simulations of the corresponding field intensities (multiplied by $\rho^{2}$ ) are shown in (d, h, l), respectively.

where $n_{\text {glass }}$ is the refractive index of the glass substrate, $\lambda$ is the wavelength of the incident light, and $\varepsilon_{\text {gold }}$ is the complex permittivity of the gold film. Taking $n_{\text {glass }}=1.525$ for the glass coverslip used as a substrate (from VWR Internation$\mathrm{al}^{29}$ ), $\lambda=633 \mathrm{~nm}$, and $\varepsilon_{\text {gold }}=-12.047+1.163 i$ (from Olmon et al..$\left.^{\circ}\right)$, we obtain $\left|\theta_{c \text {,gold }}\right|=43.3^{\circ}$.

We also perform Finite Difference Time Domain (FDTD) simulations using the Lumerical Solutions software to describe the far-field angular radiation pattern of a hollow pyramidal probe. Based on the work by Obermüller and Karrai on the free space radiation of metal coated aperture tips ${ }^{10}$, a superposition of lateral magnetic $\left(\mathrm{M}_{\mathrm{y}} \propto \mathrm{H}_{\mathrm{y}}\right)$ and electric dipoles $\left(P_{x} \propto E_{x}\right)$ with respective strengths 2 and 1 is chosen, where $x$ is the direction parallel to the incident light polarization direction. To verify the full consistency of this modeling with the experimental situations presented above, the dipoles are firstly placed perpendicular to each other in air (Figure 3.d.), then in contact with a $200 \mu \mathrm{m}$ wide transparent surface and finally on top of a $200 \mu \mathrm{m}$ wide metal-coated glass surface (Figures 3.h. and 3.l. respectively). Note that the use of dipoles in the modeling combined with FDTD simulations is only expected to provide a qualitative description of the probe as it fails to properly describe very large radiation angles ${ }^{13}$. It also neglects the details of the geometry of the metal coated aperture tip at a sub- $\lambda$ scale $^{14}$ and the subtle interaction with two plane metal-dielectric interfaces which comes into play when it is used to excite leaky surface plasmons on a metal film ${ }^{4,5}$. We note a striking similarity of the $\mathrm{x}-\mathrm{y}$ intensity profile (see Figure S1, Supporting Information) with the predictions of Drezet et al.5, which supports the relevance of this complete description. The simpler two dipole model is however adequate to qualitatively describe the experiments presented in this paper.

The electric field intensities calculated in the $\mathrm{x}-\mathrm{z}$ plane beneath the tip (Figures 3.d., 3.h. and 3.l.) show good agreement with the experimental results (Figures 3.b., 3.f. and 3.j.). Note that such an agreement could not be achieved in the three cases simultaneously if the metal-coated aperture tip was modeled either as a purely electric or as purely magnetic dipole, which clearly confirms that the probe both behaves as an electric and magnetic field source.

In addition, fringes are clearly visible in the metal layer and a few microns below it, near the top of both the experimental and calculated images, Figures 3.j. and 3.l. They are attributed to an interference between the surface plasmons and the transmitted light and/or leakage radiation ${ }^{31}$. We emphasize that a rigorous theoretical treatment of the leakage radiation produced by a horizontal electric dipole on a metal film predicts a broad nonplasmonic radiative signal which may significantly contribute to the far-field. We indeed observe a similar pattern in the experimental $x-y$ intensity profile (see Figure $S_{1}$, Supporting Information) as theoretically predicted5.

Characterization of the Angular Scattering. The ${ }_{3} \mathrm{D}$ tomographic reconstruction of the electromagnetic field in the vicinity of the NSOM tip which can be calculated from the measurement of a hologram, as described above, allows us to quantitatively describe the angular radiation patterns of the scattered light.

Figure 4 displays the far-field radiation patterns of the scattered light with the aperture tip placed in air (Figure 4.a.), and in contact with the transparent and metallic film samples (Figures 4.c. and 4.e.). The normalized intensity is plotted against the emission angle $\theta$ in Figures 4.b., 4.d. and 4.f. respectively. The experimental scatter plots (blue) are obtained by first transforming into spherical coordinates the raw data of the electromagnetic field intensity obtained from the hologram after ${ }_{3} \mathrm{D}$ reconstruction. Then, we select the values contained in the $\mathrm{x}-\mathrm{z}$ plane of the NSOM tip perpendicular to the sample surface, and in a spherical slice of thickness $\Delta \rho=30 \mathrm{~nm}$ centered on the aperture tip. This thickness is chosen in such a way that one intensity value at most is attributed to each angle $\theta$ in the resulting data selection. The black plots correspond to the simulated radiation patterns of the superposition of a $\mathrm{M}_{\mathrm{y}}$ dipole and a $\mathrm{P}_{\mathrm{x}}$ dipole for the three separate cases, and show good agreement with 
the experimental results. The vertical red lines indicate the position of the critical angle $\left|\theta_{c, \text { glass }}\right|=41.8^{\circ}$ (Figure 4.d.) and the emission angle $\left|\theta_{c, \text { gold }}\right|=43.3^{\circ}$ (Figure 4.f.). For the tip placed in free space, broad angular distribution centered around $\theta=0^{\circ}$ is observed with the intensity decaying monotonously on both sides of the maximum (Figure 4.b.). For the glass sample, we confirm that light emerges through the substrate at maximum angles of $\pm\left|\theta_{c \text {,glass }}\right|$ when the tip is in contact with the surface, in addition to forward scattering beneath the aperture tip centered around $|\theta|=0^{\circ}$ (Figure 4.d.). However, when the aperture tip is in contact with the metallic film (Figure 4.f), scattered light forms two narrow peaks at $\pm\left|\theta_{c, \text { gold }}\right|$ corresponding to the surface plasmons that are excited at the metal/air interface and tunnel through the film before leaking into the glass substrate. It occurs beyond the critical angle of a glass/air interface $\left|\theta_{\text {c,glass }}\right|=41.8^{\circ}$. Its detection is experimentally possible thanks to the high numerical aperture of the oil objective used for collection (see Measurement section).

We then carry out measurements where the tip is placed at a height of $3 \mu \mathrm{m}$ above the metallic film sample, which allows us to compare the angular radiation patterns of the scattered light when the tip is placed both in the near-field (Figures 4.e and 4.f.) and far-field regions (Figure 5). We can infer that when the tip is off-contact (Figure 5.b.), the gold film has no significant effect on the scattering angle. The maximum intensity of the far-field radiation pattern at $\rho=10 \mu \mathrm{m}$ is reduced by a factor 2.5 when the aperture tip is out of contact at a height of $3 \mu \mathrm{m}$, due to the poor transmission of the gold film. At this height, the large lateral wavevector components produced at the sub- $\lambda$ aperture tip are significantly suppressed, which hinders the efficient excitation of surface plasmons on the gold film. As a result, emission remains below $\theta_{\text {c,glass }}$ and exhibits two maxima at $\pm 40.5^{\circ}$, which is similar to the observation made when the tip is placed $3 \mu \mathrm{m}$ above the glass substrate without metallic film (not shown). These results are consistent with previous measurements by Hecht et al. ${ }^{2}$ based on the combination of back-focal plane and real space imaging. For completitude, we show in Figure S.2. (see Supporting Information) the $x-z$ section view of the wavefront reconstruction obtained experimentally when the tip is $3 \mu \mathrm{m}$ above the metallic film, for comparison with the situation where it is in contact with it (Figure 3.k). (a)

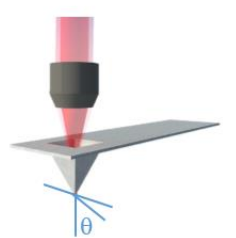

(c)

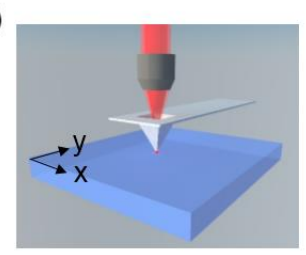

(e)

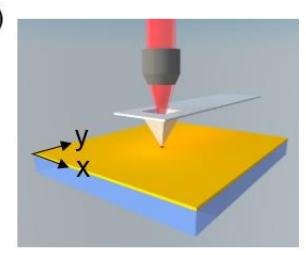

(b)

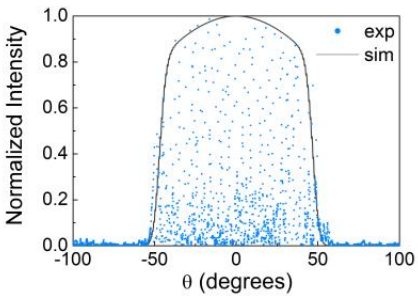

(d)

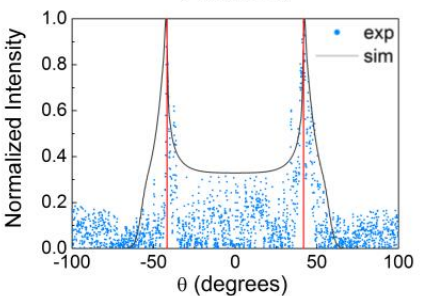

(f)

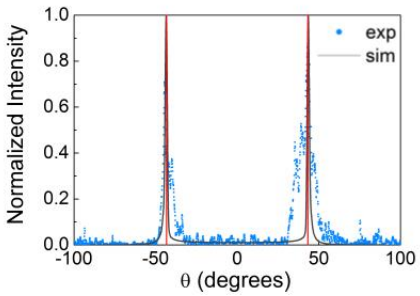

Figure 4. Far-field radiation patterns of light scattered by a NSOM tip placed in (a) free space, and in contact with (c) a transparent glass substrate, and (e) a $40 \mathrm{~nm}$ gold film on a glass substrate. $(\mathrm{b}, \mathrm{d}, \mathrm{f})$ Normalized intensity versus the emission angle $\theta$ in the $\mathrm{x}-\mathrm{z}$ plan of the NSOM tip for the three cases (a, c, e). The vertical red lines in (d) correspond to the position of the critical angle $\left|\theta_{c \text {,glass }}\right|=41.8^{\circ}$, and those in (f) correspond the emission angle $\left|\theta_{c, \text { gold }}\right|=43.3^{\circ}$. (a)

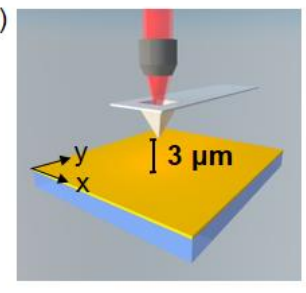

(b)

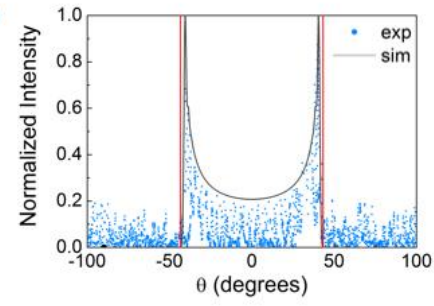

Figure 5. Far-field radiation patterns of scattered light through a $40 \mathrm{~nm}$ gold film on a glass substrate from a NSOM tip placed at $3 \mu \mathrm{m}$ above the sample surface (a). (b) Normalized intensity versus the emission angle $\theta$ in the $x-z$ plan of the NSOM tip. Experimental data are plotted in blue and FDTD simulations in black. The vertical red line corresponds to the position of the resonance angle of excitation of leaky surface plasmons $\left|\theta_{\text {c,gold }}\right|=43.3^{\circ}$.

\section{CONCLUSIONS}

In summary, a full-field off-axis holography technique adapted on a NSOM setup provides an accurate threedimensional reconstruction of light scattered from its tip in near-field interaction with the environment. After a numerical Fourier analysis of the recorded hologram, the amplitude and phase of the EM field can be calculated at any plane away from the source, which is a crucial information to fully characterize the tip and overcome the limitations of the NSOM approach. This method is ap- 
plied here to reconstruct in $3 \mathrm{D}$ a tomographic image of the scattered light from the metal-coated hollow pyramidal aperture tip of a NSOM in free space and coupled to transparent and plasmonic media. FDTD simulations show good agreement with the experimental results, and indicate that such hollow pyramidal probes can be modeled as a superposition of magnetic and electric dipoles perpendicular to each other in the plane of the aperture.

With the DHM/NSOM hybrid system, we have shown that the coupling of a NSOM tip to the environment has a dramatic influence on the way it scatters light. Combined with nanolithography and nanomanipulation techniques, NSOM provides a unique way to tune the interaction between nanostructures by performing a fine adjustment of their relative position. The high versatility of the instrument combined with DHM reconstruction methods should allow one to study in detail coupled EM resonators or complex systems involving multiple scattering events. Mapping the phase in $3 \mathrm{D}$ could also be of high interest in some of these studies, beyond using it to back-propagate numerically the EM field towards the source to obtain a $3 \mathrm{D}$ reconstruction of the EM field intensity as we have done here.

\section{METHODS}

Measurement. All measurements are performed by an optical setup composed of a Mach-Zehnder off-axis interferometer combined with a modified commercial NSOM (WITec GmbH alphazooS). A 90-10 fiber splitter is used to split the light coming from a single mode He-Ne laser ( $\mathrm{Re}-$ search Electro Optics R-32413, $\lambda=633 \mathrm{~nm}, \mathrm{P}=35 \mathrm{~mW}$ ) into the sample and reference beams. The sample beam is focused through a 20x, NA=0.4 objective on the apex of a $150 \mathrm{~nm} \mathrm{SiO}$ pyramidal aperture probe coated with $120 \mathrm{~nm}$ of $\mathrm{Al}$, with a cone angle of $\sim 70$ degrees. The sample is placed on a threeaxis stage, and the distance between the sample and the tip is controlled by a piezoelectric positioner. The scattered light is collected by a 10ox, NA $=0.9$ objective when tip is in free space, and by a 10ox, NA $=1.4$ oil objective in the presence of a sample. It is then directed to a CCD camera (Photon Focus MV-D1024E-16o-CL, sensor resolution: 1024×1024, $8 \mu \mathrm{m} \times 8$ $\mu \mathrm{m}$ pixel matrix, $0.2 \mathrm{sec}$ exposure time) for holography recordings. A polarizer and a half-wave plate are placed in the path of both the sample and reference beams to ensure that the incident light on the NSOM probe is linearly polarized and that a maximum contrast is attained. The reconstruction procedure is carried out by a fast Fourier transform (FTT) algorithm that performs a Fourier transform of the recorded hologram, allowing spatial filtering of the unwanted diffraction orders to reduce the noise, and then propagates the complex field towards the source.

Fabrication. The transparent sample is made of a $160 \mu \mathrm{m}$ glass coverslip (VWR Micro Cover Glasses, No. 1). The plasmonic sample is made of a $40 \mathrm{~nm}$ gold film evaporated on an identical glass coverslip.

Simulation. Simulations are performed using the Finite Difference Time Domain (FDTD) method (Lumerical Solutions Inc). A tangential lateral magnetic dipole $\mathrm{H}_{\mathrm{y}}$ and an electric dipole $E_{x}$ of strengths 2 and 1, respectively, are placed perpendicular to each other in air, and at various distances from a $200 \mu \mathrm{m}$ wide substrate. A frequency-domain field monitor, used to collect the field profile in the frequency domain, is placed in the $x-z$ plane at the position of the NSOM tip to record the electromagnetic intensity of the transmitted light up to a distance of $10 \mu \mathrm{m}$ below the dipole sources. Another monitor is placed exactly at $10 \mu \mathrm{m}$ below the dipoles to evaluate the far-field projection of the transmitted radiation by Fourier transform calculations. Perfectly matched layer (PML) absorbing boundary conditions (32 PML layers) that are impedance matched to the simulation region and its materials are used. The value of the complex permittivity of gold is $\varepsilon_{\text {gold }}=-12.047+1.163 i$ at $633 \mathrm{~nm}$, taken from Olmon et al. $3^{\circ}$.

\section{SUPPORTING INFORMATION}

The supporting information shows cross sections in the $x-y$ plane of the reconstructed scattered field intensity measured on the sample made of a metallic film on glass (Figure $\mathrm{S}_{1}$ ), and a $\mathrm{x}-\mathrm{z}$ section view of the wavefront reconstruction obtained when the tip is $3 \mu \mathrm{m}$ above the metallic film (Figure S2).

\section{AUTHOR INFORMATION}

\section{Corresponding Authors}

* Emails: yannick.dewilde@espci.fr, gilles.tessier@parisdescartes.fr

\section{ACKNOWLEDGMENT}

This research was supported by LABEX WIFI (Laboratory of Excellence within the French Program Investments for the Future) under references ANR-10-LABX-24 and ANR-10IDEX-ooo1-02 PSL*, and Agence Nationale de la Recherche (ANR), Project CarISOVERRE, ANR-16-CEo9-0o12.

The authors thank Mathias Fink, Arthur Goetschy and Roman Pierrat, from the Institut Langevin for stimulating discussions and constructive comments.

\section{REFERENCES}

(1) Pohl, D. W.; Denk, W.; Lanz, M. Optical Stethoscopy: Image Recording with Resolution $\lambda / 20$. Appl. Phys. Lett. 1984, 44 (7), 651-653.

(2) Hecht, B.; Bielefeldt, H.; Novotny, L.; Inouye, Y.; Pohl, D. W. Local Excitation, Scattering, and Interference of Surface Plasmons. Phys. Rev. Lett. 1996, 77 (9), 1889-1892.

(3) Bharadwaj, P.; Bouhelier, A.; Novotny, L. Electrical Excitation of Surface Plasmons. Phys. Rev. Lett. 2011, 106 (22), 226802.

(4) Marty, R.; Girard, C.; Arbouet, A.; Colas des Francs, G. Near-Field Coupling of a Point-like Dipolar Source with a Thin Metallic Film: Implication for STM Plasmon Excitations. Chem. Phys. Lett. 2012, 532, 100-105.

(5) Drezet, A.; Genet, C. Imaging Surface Plasmons: From Leaky Waves to Far-Field Radiation. Phys. Rev. Lett. 2013, 110 (21), 213901.

(6) Novotny, L.; Hecht, B. Principles of Nano-Optics; Cambridge university press, 2012.

(7) Sánchez, E. J.; Novotny, L.; Xie, X. S. Near-Field Fluorescence Microscopy Based on Two-Photon Excitation with Metal Tips. Phys. Rev. Lett. 1999, 82 (20), 4014.

(8) Greffet, J.-J.; Carminati, R. Image Formation in nearField Optics. Prog. Surf. Sci. 1997, 56 (3), 133-237. 
(9) Bethe, H. A. Theory of Diffraction by Small Holes. Phys. Rev. 1944, 66 (7-8), 163.

(10) Obermüller, C.; Karrai, K. Far Field Characterization of Diffracting Circular Apertures. Appl. Phys. Lett. 1995, 67 (23), 3408-3410.

(11) Obermüller, C.; Karrai, K.; Kolb, G.; Abstreiter, G. Transmitted Radiation through a Subwavelength-Sized Tapered Optical Fiber Tip. Ultramicroscopy 1995, 61 (1), 171-177.

(12) Drezet, A.; Woehl, J. C.; Huant, S. Diffraction by a Small Aperture in Conical Geometry: Application to MetalCoated Tips Used in near-Field Scanning Optical Microscopy. Phys. Rev. E 2002, 65 (4).

(13) Drezet, A.; Woehl, J. C.; Huant, S. Extension of Bethe's Diffraction Model to Conical Geometry: Application to nearField Optics. Europhys. Lett. 20o1, 54 (6), 736.

(14) Drezet, A.; Cuche, A.; Huant, S. Near-Field Microscopy with a Single-Photon Point-like Emitter: Resolution versus the Aperture Tip? Opt. Commun. 2011, 284 (5), 1444-1450.

(15) Denkova, D.; Verellen, N.; Silhanek, A. V.; Valev, V. K.; Dorpe, P. V.; Moshchalkov, V. V. Mapping Magnetic Near-Field Distributions of Plasmonic Nanoantennas. ACS Nano 2013, 7 (4), 3168-3176.

(16) Curto, A. G.; Volpe, G.; Taminiau, T. H.; Kreuzer, M. P.; Quidant, R.; Hulst, N. F. van. Unidirectional Emission of a Quantum Dot Coupled to a Nanoantenna. Science 2010, 329 (5994), 930-933.

(17) Xie, Z.; Lefier, Y.; Suarez, M. A.; Mivelle, M.; Salut, R.; Merolla, J.-M.; Grosjean, T. Doubly Resonant Photonic Antenna for Single Infrared Quantum Dot Imaging at Telecommunication Wavelengths. Nano Lett. 2017, 17 (4), 2152-2158.

(18) Thomas, M.; Greffet, J. J.; Carminati, R.; AriasGonzalez, J. R. Single-Molecule Spontaneous Emission close to Absorbing Nanostructures. Appl. Phys. Lett. 2004, 85 (17), 38633865 .

(19) Babuty, A.; Joulain, K.; Chapuis, P.-O.; Greffet, J.-J.; De Wilde, Y. Blackbody Spectrum Revisited in the Near Field. Phys. Rev. Lett. 2013, 110 (14), 146103.

(20) Joulain, K.; Ben-Abdallah, P.; Chapuis, P.-O.; De Wilde, Y.; Babuty, A.; Henkel, C. Strong Tip-sample Coupling in Ther- mal Radiation Scanning Tunneling Microscopy. J. Quant. Spectrosc. Radiat. Transf. 2014, 136 (Supplement C), 1-15.

(21) Drezet, A.; Hohenau, A.; Koller, D.; Stepanov, A.; Ditlbacher, H.; Steinberger, B.; Aussenegg, F. R.; Leitner, A.; Krenn, J. R. Leakage Radiation Microscopy of Surface Plasmon Polaritons. Mater. Sci. Eng. B 2008, 149 (3), 220-229.

(22) Berthel, M.; Jiang, Q.; Pham, A.; Bellessa, J.; Genet, C.; Huant, S.; Drezet, A. Directional Local Density of States of Classical and Quantum Propagating Surface Plasmons. Phys. Rev. Appl. 2017, 7 (1), 14021.

(23) Lieb, M. A.; Zavislan, J. M.; Novotny, L. SingleMolecule Orientations Determined by Direct Emission Pattern Imaging. JOSA $B$ 2004, 21 (6), 1210-1215.

(24) Cuche, E.; Marquet, P.; Depeursinge, C. Spatial Filtering for Zero-Order and Twin-Image Elimination in Digital offAxis Holography. Appl. Opt. 20oo, 39 (23), 4070-4075.

(25) Suck, S. Y.; Collin, S.; Bardou, N.; De Wilde, Y.; Tessier, G. Imaging the Three-Dimensional Scattering Pattern of Plasmonic Nanodisk Chains by Digital Heterodyne Holography. Opt. Lett. 2011, 36 (6), 849-851.

(26) Park, C.; Park, J.-H.; Rodriguez, C.; Yu, H.; Kim, M.; Jin, K.; Han, S.; Shin, J.; Ko, S. H.; Nam, K. T.; et al. Full-Field Subwavelength Imaging Using a Scattering Superlens. Phys. Rev. Lett. 2014, 113 (11).

(27) Parigi, V.; Perros, E.; Binard, G.; Bourdillon, C.; Maître, A.; Carminati, R.; Krachmalnicoff, V.; Wilde, Y. D. Near-Field to Far-Field Characterization of Speckle Patterns Generated by Disordered Nanomaterials. Opt. Express 2016, 24 (7), 7019-7027.

(28) Witec GmbH www.witec.de.

(29) VWR International - Chemicals and Laboratory Scientific Supplies www.vwr.com.

(30) Olmon, R. L.; Slovick, B.; Johnson, T. W.; Shelton, D.; Oh, S.-H.; Boreman, G. D.; Raschke, M. B. Optical Dielectric Function of Gold. Phys. Rev. B 2012, 86 (23), 235147.

(31) Wang, B.; Aigouy, L.; Bourhis, E.; Gierak, J.; Hugonin, J. P.; Lalanne, P. Efficient Generation of Surface Plasmon by Single-Nanoslit Illumination under Highly Oblique Incidence. Appl. Phys. Lett. 2009, 94 (1), 11114. 

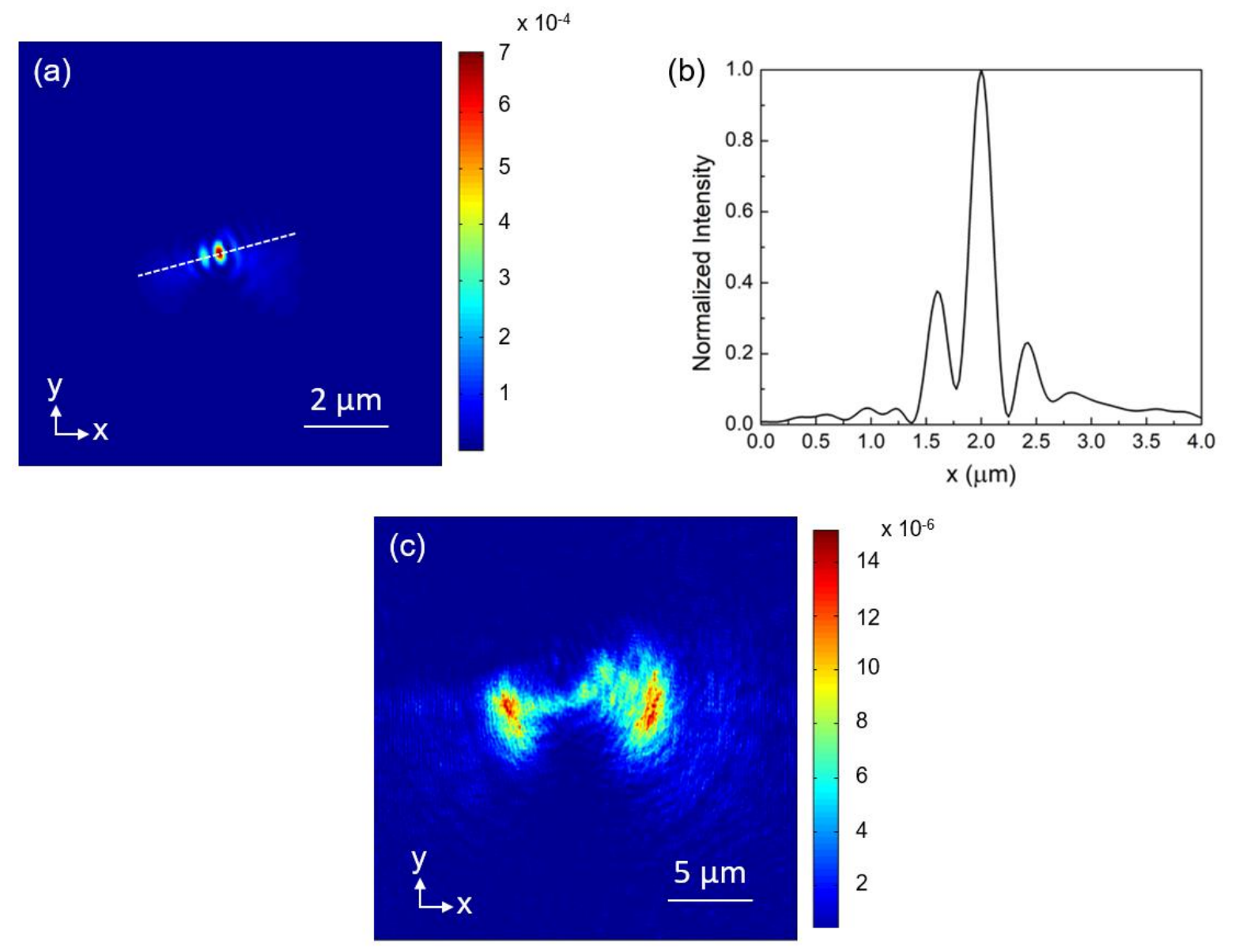

Figure S.1.: (a) 2D cross section in the $x-y$ plane at the level of the metallic film (at $z=0$ ) of the reconstructed scattered field intensity obtained by off-axis Digital Holographic Microscopy measurement of the complex EM field with the tip in contact with a metallic film on glass. (b) Section view of the 2D cross section showing the intensity profile along the dashed line in (a). (c) $2 D$ cross section in the $x-y$ plane $5 \mu \mathrm{m}$ below the metallic film (i.e. in the glass substrate at $z=-5 \mu \mathrm{m}$ ) of the reconstructed scattered field intensity measured with the tip in contact with a metallic film on glass. 


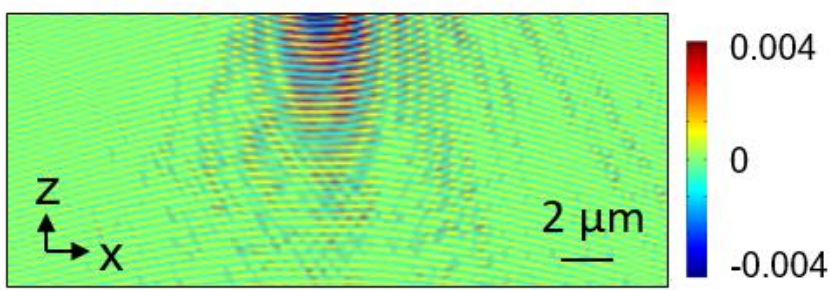

Figure S.2.: $x$-z section view of the wavefront reconstruction obtained experimentally by off-axis Digital Holographic Microscopy measurement of the complex EM field when the tip is $3 \mu \mathrm{m}$ above the metallic film, for comparison with the situation where it is in contact with it (see Figure 3.k). The figure shows $\rho|A(x, y, z)| \cos (\phi(x, y, z))$. 